\title{
Comparison of the impact of applications of Targeted Transfusion Protocol and Massive Transfusion Protocol in trauma patients
}

\author{
Shahram Paydar ${ }^{1,2}$, Hosseinali Khalilili, ${ }^{1,3}$, Golnar Sabetian ${ }^{4}$, \\ Behnam Dalfardi, ${ }^{5,6}$, Shahram Bolandparvaz ${ }^{1,2}$, Mohammad Hadi Niakan ${ }^{1,2}$, \\ Hamidreza Abbasi ${ }^{1,2}$, and Donat R. Spahn ${ }^{7}$ \\ ${ }^{1}$ Trauma Research Center, Shahid Rajaee (Emtiaz) Trauma Hospital, Departments of ${ }^{2}$ General Surgery, \\ ${ }^{3}$ Neurosurgery, ${ }^{4}$ Anesthesiology and Critical Care Research Center, ${ }^{5}$ Student Research Committee, ${ }^{6}$ Internal \\ Medicine, Shiraz University of Medical Sciences, Shiraz, Iran, ${ }^{7}$ Institute of Anesthesiology, University and University \\ Hospital of Zurich, Zurich, Switzerland
}

Background: The current study assessed a recently developed resuscitation protocol for bleeding trauma patients called the Targeted Transfusion Protocol (TTP) and compared its results with those of the standard Massive Transfusion Protocol (MTP).

Methods: Per capita utilization of blood products such as packed red blood cells (RBCs), fresh frozen plasma (FFP), and platelet concentrates was compared along with mortality rates during two 6-month periods, one in 2011 (when the standard MTP was followed) and another in 2014 (when the TTP was used). In the TTP, patients were categorized into three groups based on the presence of head injuries, long bone fractures, or penetrating injuries involving the trunk, extremities, or neck who were resuscitated according to separate algorithms. All cases had experienced motor vehicle accidents and had injury severity scores over 16 .

Results: No statistically significant differences were observed between the study groups at hospital admission. Per capita utilization of RBC ( $4.76 \pm 0.92$ vs. $3.37 \pm 0.55$; $\mathrm{P}=0.037)$, FFP ( $3.71 \pm 1.00$ vs. $2.40 \pm 0.52$; $\mathrm{P}=0.025)$, and platelet concentrate $(1.18 \pm 0.30$ vs. $0.55 \pm 0.18 ; \mathrm{P}=0.006)$ blood products were significantly lower in the TTP epoch. Mortality rates were similar between the two study periods $(\mathrm{P}=0.74)$.

Conclusions: Introduction of the TTP reduced the requirements for RBCs, FFP, and platelet concentrates in severely injured trauma patients.

Key Words: Guidelines, Mortality, Transfusion, Trauma, Wounds and injuries.

Corresponding author: Behnam Dalfardi, M.D.

Department of Internal Medicine, Shiraz University of Medical Sciences, Namazee Hospital, Namazee Square, Zand Street, Shiraz 71937-11351, Iran

Tel: 98-9132483359, Fax: 98-71336254206, Email: Dalfardibeh@gmail.com

ORCID: https://orcid.org/0000-0003-4547-0965

Received: February 10, 2017. Revised: April 17, 2017 (1st); May 5, 2017 (2nd). Accepted: May 11, 2017.

Korean J Anesthesiol 2017 December 70(6): 626-632

https://doi.org/10.4097/kjae.2017.70.6.626

(c) This is an open-access article distributed under the terms of the Creative Commons Attribution Non-Commercial License (http://creativecommons.org/ licenses/by-nc/4.0/), which permits unrestricted non-commercial use, distribution, and reproduction in any medium, provided the original work is properly cited. 


\section{Introduction}

Trauma remains a major cause of mortality worldwide [1] According to the available data, uncontrolled hemorrhage is responsible for about $40 \%$ of potentially preventable deaths following trauma, particularly in the first 24 hours post-injury [2,3]. Therefore, early identification of bleeding sources, efficacious hemorrhage control, and prompt resuscitation are needed to improve the outcome of trauma patients $[2,4]$.

Uncontrolled post-traumatic bleeding frequently leads to a lethal triad of coagulopathy, hypothermia, and acidosis, events that further complicate resuscitation and operative control of hemorrhage [4-6]. The condition of each trauma case determines the individual need for specific blood products, including packed red blood cells (RBCs), fresh frozen plasma (FFP), and platelet concentrates (PCs), to treat hypovolemia due to hemorrhage. It is obvious that the use of these blood products in clinical practice should follow predetermined rules [7-9].

Parallel to improved understanding of the pathophysiology of trauma and hemorrhagic shock, the strategies used to manage these conditions have evolved over time [9]. Presently, standard massive transfusion protocols (MTPs) are most commonly used to resuscitate bleeding trauma patients in trauma centers $[10,11]$. However, various studies have demonstrated conflicting results regarding the impact of these protocols on clinical outcomes [12-18]. In addition, the use of blood products in trauma cases can be risky. For example, despite the benefits of FFP in trauma patients with coagulopathy, some studies have shown adverse effects of it, including volume overload, acute lung injury, and nosocomial infections [18].

These issues have led to current, ongoing discussions about the ideal resuscitation strategy, and developing trauma resuscitation guidelines still remains a constant concern for trauma personnel. The trauma team at Shahid Rajaee Hospital, a highturnover level-one trauma hospital affiliated with the Shiraz University of Medical Sciences, Shiraz, Iran, recently developed local algorithms for the resuscitation of bleeding trauma patients called the Targeted Transfusion Protocol (TTP). The goal of this single-center study was to compare the TTP algorithm with the previously used MTP guidelines.

\section{Materials and Methods}

\section{Study protocol}

This study was performed at Shahid Rajaee Trauma Center, a level-one main-referral trauma hospital in southern Iran, which is affiliated with the Shiraz University of Medical Sciences. The study protocol was approved by Iran's Ministry of Health and Medical Education (code: HMD/137; date: April 21, 2014) and
Table 1. The Criteria for Patient Referral to the Resuscitation Room

GCS $\leq 10$

Systolic blood pressure $\leq 90 \mathrm{mmHg}$

2 or more long bone fractures

Respiratory distress

Major organ failure (liver failure, renal failure, etc.)

These criteria were the same in both study periods. GCS: glasgow coma scale.

the Shiraz University of Medical Sciences' Institutional Review Board. This study adhered to the Ethical Principles for Medical Research Involving Human Subjects, outlined in the Helsinki Declaration of 1975 (revised in 2000).

This study compared the numbers of RBC, FFP, and PC transfusions utilized during the 6-month period from the beginning of May to the end of October in 2011 (MTP) and again in 2014 (TTP). It should be noted that we did not use FVIIa in our center (due to its high cost and our limited resources). Trauma patients referred to the Shahid Rajaee Trauma Center's resuscitation room (Table 1) were prospectively included in this study.

A general practitioner under the supervision of an emergency medicine specialist was responsible for referring patients to the resuscitation room. In the hospital's resuscitation room, the general surgery team instigated either the standard MTP (in 2011) or TTP (in 2014). Notably, in both protocols, resuscitation was initiated using $2 \mathrm{~L}$ of crystalloids, after which blood products were transfused according to the protocol. The maximum amount of crystalloid transfusion was $3.5 \mathrm{~L}$ per day.

All blood samples were collected after patients entered the resuscitation room. The general surgery resident followed up and interpreted the laboratory results.

The neurosurgery team was actively involved in the management of head injury patients. A loss of consciousness or amnesia after injury, a Glasgow Coma Scale (GCS) score of less than 10, or clinical findings to suggest a skull bone fracture were considered to indicate a head injury. Such patients, just after stabilization of vital signs, underwent brain computed tomography (CT) scans. Those cases suspected of having a head injury whose vital signs did not stabilize (with a heart rate elevated over 120 bpm) after resuscitation with crystalloids and appropriate pain control were sent for brain CT scans accompanied by a general surgery resident who simultaneously performed resuscitation techniques.

\section{Definitions}

The following indices were used to compare blood products used during the two study time frames (based on the data from the entire hospital stay period):

- Per capita requisition: the mean value of blood product 
requisition per patient during each one-month period. It should be noted that, in the standard MTP, blood products are prepared (and requested) in the form of shipments. However, in some cases, such as with an adequate response to resuscitation measures, all of these requested products may not be used and may end up being wasted.

- Per capita consumption: the mean value of blood product consumption per patient during each one-month period.

\section{Shahid Rajaee Hospital trauma resuscitation guidelines}

In 2011, the Shahid Rajaee Trauma Center followed a standard MTP for resuscitation of trauma patients (Fig. 1); however, in 2014 , local guidelines for trauma resuscitation (Figs. 2-4) were followed.

In accordance with the Shahid Rajaee Trauma Hospital local guidelines, patients were categorized into three groups:

1. Cases of multiple trauma without concomitant head injury.

2. Cases of multiple trauma with concomitant head injury.

3. Cases of penetrating trauma involving the trunk, extremities, or neck without concomitant head injury.

Patients meeting the above-mentioned criteria were resuscitated according to distinct guidelines (Figs. 2-4).
Additionally, fibrinogen has been used to resuscitate trauma patients with a fibrinogen level of less than $100 \mathrm{mg} / \mathrm{dl}$ since 2012.

\section{Statistical analysis}

Data were analyzed using descriptive statistics and the T-test (SPSS Statistics Software, version 16; Chicago, IL, USA). A P value of less than 0.05 was considered statistically significant.

\section{Results}

During the standard MTP study period in 2011, a total of 957 trauma patients were referred to Shahid Rajaee Trauma Center's resuscitation room. In the TTP period of 2014, a total of 1094 patients were referred to Shahid Rajaee Trauma Center's resuscitation room. All study participants had experienced a motor vehicle accident and had an injury severity score (ISS) of higher than 16

There was no significant difference in the mean total number of admitted patients and the mean total mortality rates between the two study periods. Mortality status was compared between two groups using chi-square test (Table 2). However, the per capita utilization of RBC $(4.76 \pm 0.92$ [mean total in 2011] vs.

- Intravenous access (2 large IVs)

Initial measures

- Sampling (blood group and Rh, cross match, ABG, CBC, PT, PTT, INR, electrolytes)

- Initiate hydration with crystalloids; if the patient remained hemodynamically unstable after $2 \mathrm{~L}$, switch to RBC

- Establish normothermia

- Exclude ongoing surgical blood loss

Criteria for massive transfusion

- 6 or more units of RBC in one bleeding episode with ongoing losses

- 4 or more units of RBC within one hour with ongoing losses

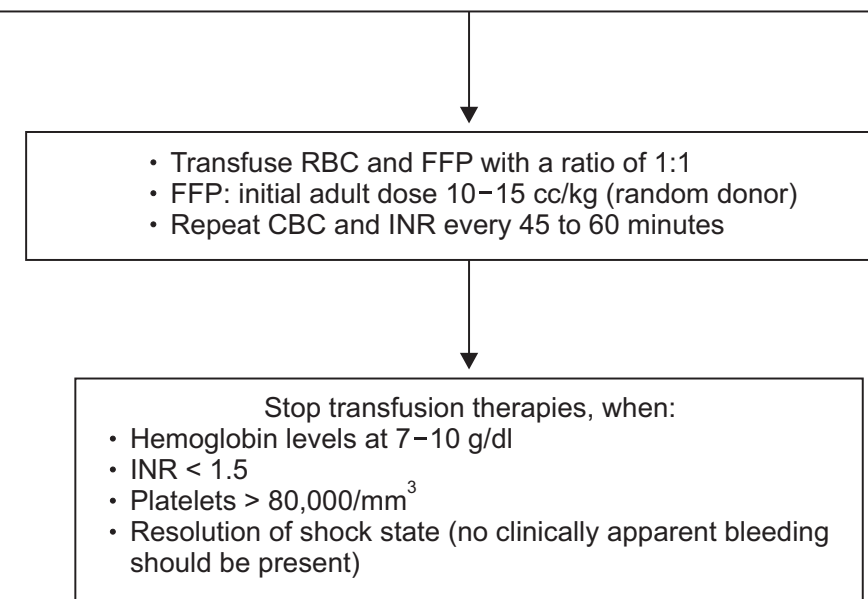




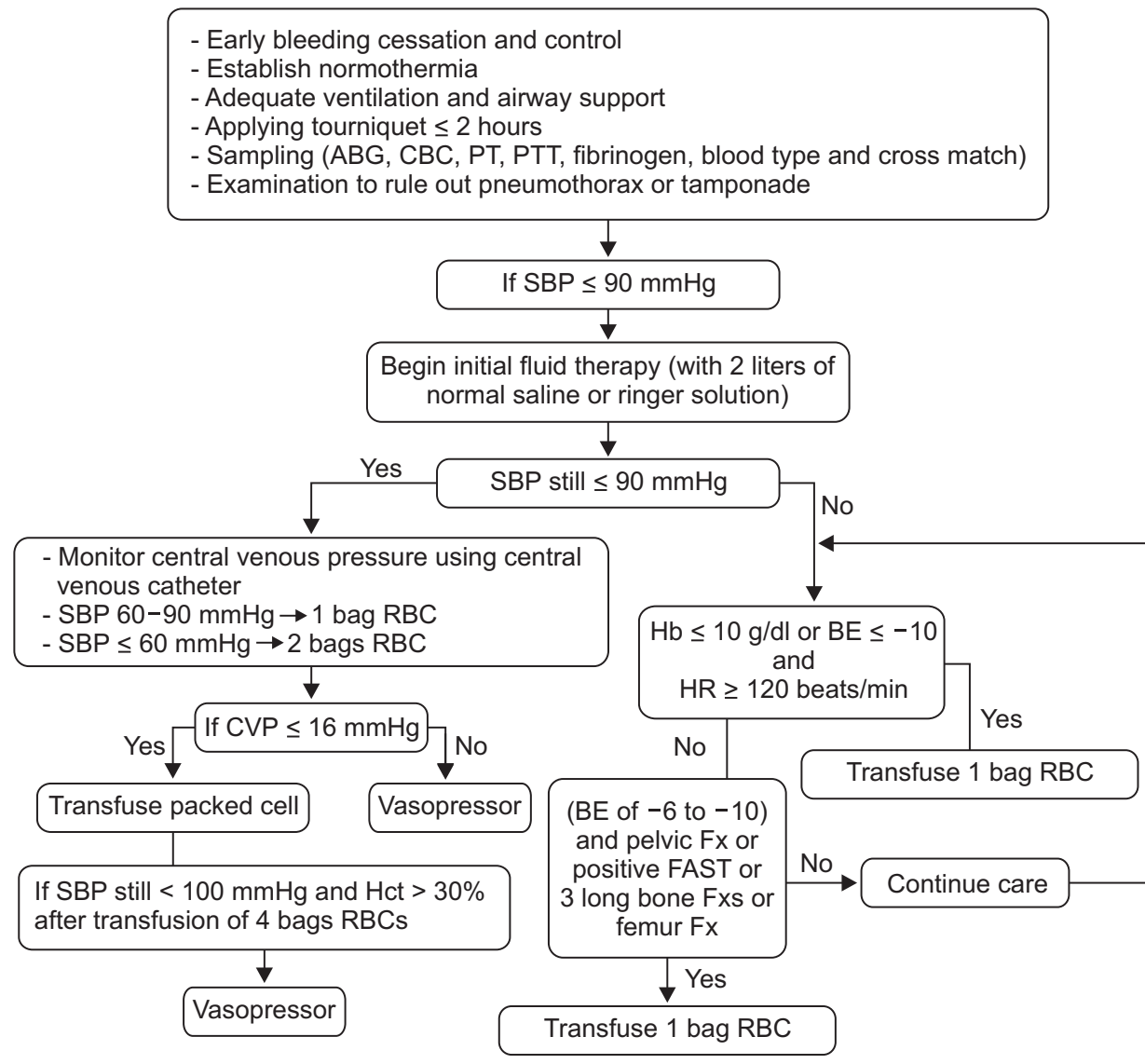

Fig. 2. Algorithm for blood product transfusions in multiple trauma patients without traumatic brain injury. ABG: arterial blood gas, CBC: complete blood count, PT: prothrombin time, PTT: partial thromboplastin time, SBP: systolic blood pressure, RBC: red blood cell, Hb: hemoglobin, BE: base excess, HR: heart rate, CVP: central venous pressure, FAST: focused assessment with sonography in trauma, Fx: fracture.
$3.37 \pm 0.55$ [mean total in 2014]; $\mathrm{P}=0.037)$, FFP $(3.71 \pm 1.00$ vs. $2.40 \pm 0.52 ; \mathrm{P}=0.025)$, and PCs ( $1.18 \pm 0.30$ vs. $0.55 \pm 0.18 ; \mathrm{P}=$ $0.006)$ were significantly lower in the TTP epoch. The mortality rate was similar between the two study periods $(\mathrm{P}=0.74)$. In addition, near-significant reductions in the per capita requisition of PC and per capita consumption of FFP were observed in this study.

The mean durations of hospital stay were similar in 2011 and 2014 (19 days for both periods). The mean ICU stay duration was 10.5 days in 2011 and 9 days in 2014.

\section{Discussion}

The results of the current study indicate that the introduction of TTP guidelines into clinical practice led to a statistically significant reduction in per capita utilization of RBC, FFP, and PC blood products compared with the use of a standard MTP. No impact on mortality was found.

Presently, the standard MTP is a blood product transfusion guideline widely used in trauma centers worldwide [10,11]. This guideline was developed to ensure appropriate communication and coordination between medical services, including trauma and nursing teams, involved in the care of trauma patients who require a transfusion. The protocol provides an algorithmic approach to the transfusion of blood products and required laboratory studies in order to facilitate clinical care [19]. We developed new guidelines in order to reduce the quantity of blood products utilized. The current study indicated that clinical introduction of TTP algorithms successfully reduced the use of blood products; however, no change in mortality was observed.

Previous studies have shown that blood product transfusions used to resuscitate bleeding trauma patients may have some adverse effects. For example, Chaiwat et al. [20] found early RBC transfusions in trauma patients to be an independent risk factor for acute respiratory distress syndrome (ARDS) with a $6 \%$ increase in the risk of ARDS for each RBC transfusion. Accordingly, they recommended following a conservative $\mathrm{RBC}$ transfusion policy in trauma cases [20]. Another study by Acker et al. [21] reported an increased risk of death in children with traumatic brain injury following RBC transfusion. It has also been suggested that allogeneic blood transfusion in surgical patients is a major risk factor for the development of post-operative bacterial infection [22]. This risk factor is more significant in trauma cases than in elective surgical patients [22]. Adverse effects are also possible following the use of other blood products, such as FFP or PCs. For instance, they may cause transfusion-related 


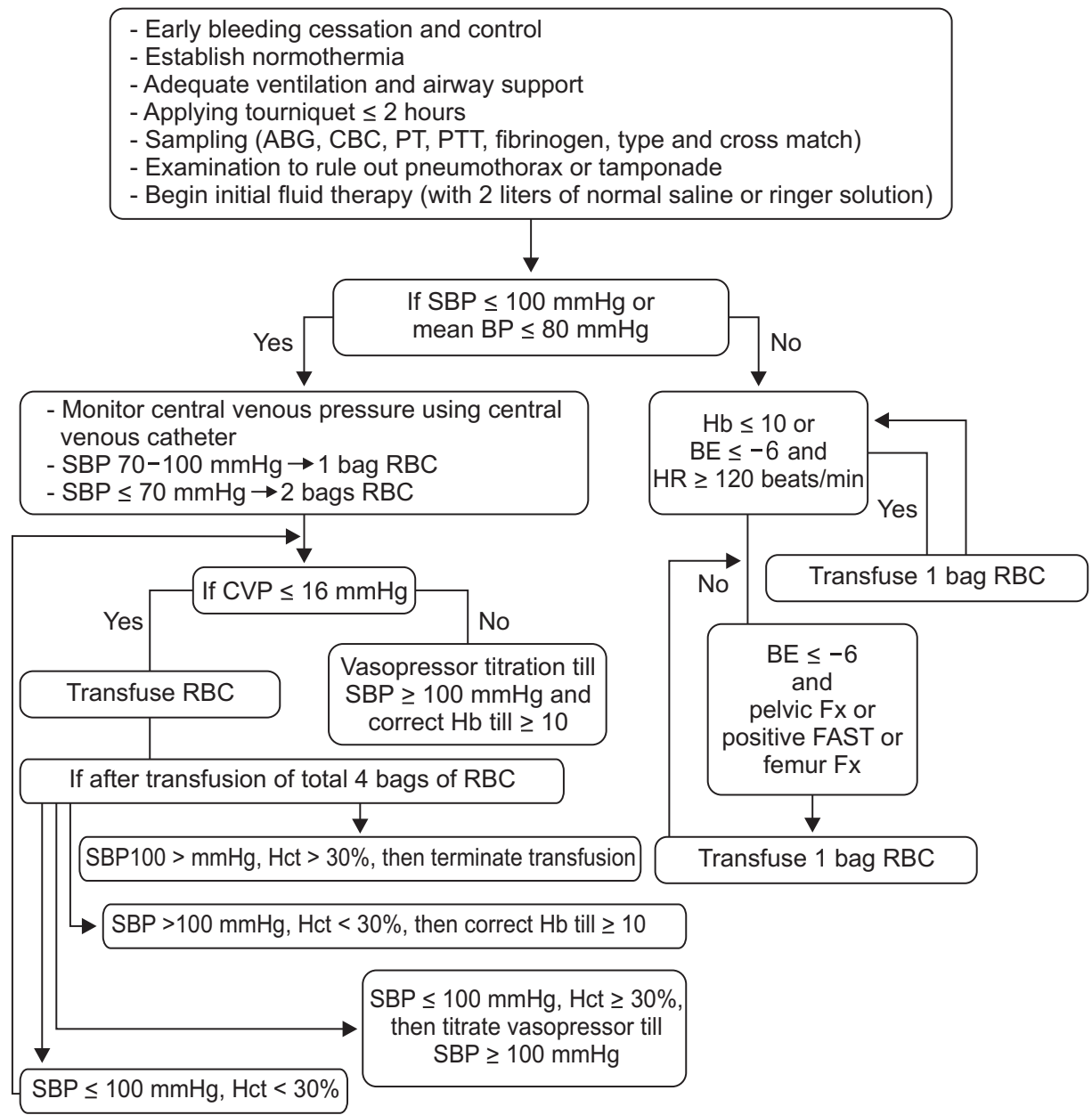

Fig. 3. Algorithm for blood product transfusions in multiple trauma patients with traumatic brain injury. ABG: arterial blood gas, CBC: complete blood count, PT: prothrombin time, PTT: partial thromboplastin time, SBP: systolic blood pressure, $\mathrm{Hb}$ : hemoglobin ( $\mathrm{g} / \mathrm{dl}$ ), $\mathrm{BE}$ : base excess, RBC: red blood cell, HR: heart rate, CVP: central venous pressure, Hct: hematocrit (\%), Fix: fracture, FAST: focused assessment with sonography in trauma.

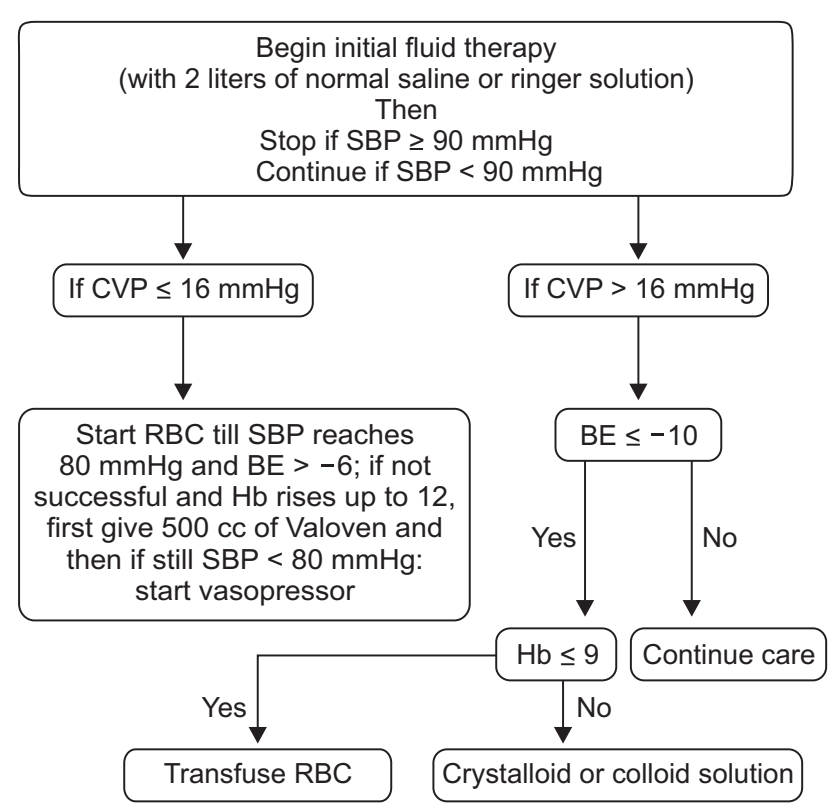

Fig. 4. Algorithm for blood product transfusions in cases of penetrating trauma of the trunk, extremities, or neck without traumatic brain injury. BP: blood pressure, SBP: systolic blood pressure, CVP: central venous pressure, RBC: red blood cell, Hb: hemoglobin (g/dl), BE: base excess. acute lung injury (TRALI) [23]. Such adverse consequences were a main factor in the authors' decision to develop new algorithms for the management of bleeding trauma patients. According to this study, the TTP protocol can efficiently reduce the use of perioperative blood products, which helps to prevent transfusion-related complications, particularly TRALI [24].

One important aspect of any newly developed medical protocol is its cost-benefit ratio in the health-care system, which is more significant in centers with high admission numbers and high turn-over, such as the Shahid Rajaee Trauma Center. The U.S. Department of Health and Human Services' 2011 National Blood Collection and Utilization Survey Report indicated that, in 2011, hospitals paid an average of $\$ 225.42$ per unit of leukocyte-filtered red cells, $\$ 57.91$ per unit of plasma (frozen within 8 hours of phlebotomy), $\$ 535.17$ per unit of apheresis platelets (leukocyte-reduced), and $\$ 62.41$ per unit of cryoprecipitate [25]. The present study showed that clinical introduction of TTP algorithms can reduce costs by reducing the use of blood products. It should be noted that there was no difference between the standard MTP and TTP algorithms regarding the types of laboratory examinations, including coagulation, hema- 
Table 2. Per Capita Consumption/requisition of Blood Products

\begin{tabular}{|c|c|c|c|c|c|c|c|c|}
\hline \multirow{2}{*}{ Date } & \multicolumn{3}{|c|}{ Per capita consumption } & \multicolumn{3}{|c|}{ Per capita requisition } & \multirow{2}{*}{$\begin{array}{l}\text { Number of } \\
\text { mortalities }\end{array}$} & \multirow{2}{*}{ ISS $>16^{\dagger}$} \\
\hline & $\mathrm{RBC}$ & FFP & PC & $\mathrm{RBC}$ & FFP & PC & & \\
\hline Mean Total - $2011\left(95 \% \mathrm{CI}^{\S}\right)$ & $\begin{array}{c}4.76 \\
(4.02 \text { to } 5.50)\end{array}$ & $\begin{array}{c}3.37 \\
\text { (2.61 to } 4.13)\end{array}$ & $\begin{array}{c}1.18 \\
\text { (0.94 to } 1.42)\end{array}$ & $\begin{array}{c}7.70 \\
\text { (6.8 to } 8.60 \text { ) }\end{array}$ & $\begin{array}{c}3.71 \\
\text { (2.91 to } 4.51)\end{array}$ & $\begin{array}{c}1.29 \\
(0.99 \text { to } 1.59)\end{array}$ & $\begin{array}{c}30.50 \\
(24.29 \text { to } 36.71)\end{array}$ & 159.50 \\
\hline Mean Total - 2014 (95\% CI) & $\begin{array}{c}3.37 \\
\text { (2.93 to } 3.81)\end{array}$ & $\begin{array}{c}2.35 \\
\text { (1.95 to } 2.75)\end{array}$ & $\begin{array}{c}0.55 \\
(0.41 \text { to } 0.69)\end{array}$ & $\begin{array}{c}6.56 \\
\text { (5.83 to } 7.29)\end{array}$ & $\begin{array}{c}2.40 \\
\text { (1.98 to } 2.82)\end{array}$ & $\begin{array}{c}0.97 \\
\text { (0.09 to } 1.85)\end{array}$ & $\begin{array}{c}31.66 \\
(26.08 \text { to } 37.24)\end{array}$ & 182.33 \\
\hline P value * & $0.037^{\ddagger}$ & 0.055 & $0.006^{\ddagger}$ & 0.055 & $0.025^{\ddagger}$ & 0.078 & 0.748 & 0.055 \\
\hline
\end{tabular}

RBC: red blood cells, FFP: fresh frozen plasma, PC: platelet concentrate, Mean Total: mean values of cumulative data. ${ }^{*} \mathrm{P}$ value: the significance of the difference between mean totals in 2011 and $2014 .{ }^{\dagger}$ Number of admitted patients with an injury severity score (ISS) $>16$ per month. ${ }^{7}$ Indicates a statistically significant difference (P value < 0.05). ${ }^{\S} 95 \%$ CI: $95 \%$ confidence interval.

tology, and blood gas tests. Another important point is that the TTP protocol resulted in the preparation of a reduced number of blood products by blood bank personnel, which lowered the blood bank workload. Nevertheless, further studies are required to explore these findings more comprehensively.

It should be noted that, during the study period from 2011 to 2014, there were no changes to the hospital's management system or medical facilities (except a change in resuscitation protocols) that could affect resuscitation measures or patient outcomes.

This study had some limitations. First, it did not compare possible changes in the amount of other types of blood products (such as cryoprecipitate and fibrinogen) that are used. Secondly, it would have been beneficial to compare the incidence of adverse effects (such as ARDS and nosocomial infection) in the two study periods. However, it should be noted that, in multiple injury patients, particularly those cases with a higher ISS, it is sometimes difficult to discriminate conclusively between transfusion-related morbidities and those morbidities that resulted from the traumatic event (for instance, TRALI versus lung contusion, fat emboli, or acute lung infections). Third, data on coagulation profiles were not compared between the two groups; this point should be considered in future studies. Another noteworthy issue of the current study is that we did not categorize the results based on the type of damage (head injury, long bone fracture, or penetrating injury). The consideration of this point in future studies will provide more detailed data regarding the benefits or pitfalls of the TTP protocol. Finally, as previously mentioned, further studies are needed to assess the effects of the TTP (compared with the standard MTP) on various costs that a health-care system and its patients may face.

In summary, the current study showed that TTP algorithms can significantly reduce the amount of blood products utilized compared to the standard MTP. However, further studies are necessary to examine various aspects of the clinical usage of the TTP protocol, including its cost-benefit ratio for health-care systems.

\section{ORCID}

Shahram Paydar, https://orcid.org/0000-0002-6980-2576

Hosseinali Khalili, https://orcid.org/0000-0002-3390-4212

Golnar Sabetian, https://orcid.org/0000-0001-8764-2150

Behnam Dalfardi, https://orcid.org/0000-0003-4547-0965

Shahram Bolandparvaz, https://orcid.org/0000-0002-8875-5998

Mohammad Hadi Niakan, https://orcid.org/0000-0003-0986-2187

Hamidreza Abbasi, https://orcid.org/0000-0002-8632-7084

Donat R. Spahn, https://orcid.org/0000-0002-4782-183X

\section{References}

1. Paydar S, Ahmadi A, Dalfardi B, Shakibafard A, Abbasi H, Bolandparvaz S. Clinical and economic effects of selective radiological evaluation of high-energy trauma patients: a prospective experience of a level 1 busy trauma centre. Emerg Med J 2015; 32: 535-8.

2. Kauvar DS, Lefering R, Wade CE. Impact of hemorrhage on trauma outcome: an overview of epidemiology, clinical presentations, and therapeutic considerations. J Trauma 2006; 60(6 Suppl): S3-11.

3. Khoshmohabat H, Dalfardi B, Dehghanian A, Rasouli HR, Mortazavi SM, Paydar S. The effect of CoolClot hemostatic agent on skin wound healing in rats. J Surg Res 2016; 200: 732-7.

4. Holcomb JB, Jenkins D, Rhee P, Johannigman J, Mahoney P, Mehta S, et al. Damage control resuscitation: directly addressing the early coagulopathy of trauma. J Trauma 2007; 62: 307-10.

5. Brohi K, Cohen MJ, Ganter MT, Schultz MJ, Levi M, Mackersie RC, et al. Acute coagulopathy of trauma: hypoperfusion induces systemic anticoagulation and hyperfibrinolysis. J Trauma 2008; 64: 1211-7. 
6. Tapia NM, Chang A, Norman M, Welsh F, Scott B, Wall MJ Jr, et al. TEG-guided resuscitation is superior to standardized MTP resuscitation in massively transfused penetrating trauma patients. J Trauma Acute Care Surg 2013; 74: 378-85.

7. Tien H, Nascimento B Jr, Callum J, Rizoli S. An approach to transfusion and hemorrhage in trauma: current perspectives on restrictive transfusion strategies. Can J Surg 2007; 50: 202-9.

8. Borgman MA, Spinella PC, Perkins JG, Grathwohl KW, Repine T, Beekley AC, et al. The ratio of blood products transfused affects mortality in patients receiving massive transfusions at a combat support hospital. J Trauma 2007; 63: 805-13.

9. Spinella PC, Holcomb JB. Resuscitation and transfusion principles for traumatic hemorrhagic shock. Blood Rev 2009; $23: 231-40$.

10. Young PP, Cotton BA, Goodnough LT. Massive transfusion protocols for patients with substantial hemorrhage. Transfus Med Rev 2011; 25: 293-303.

11. Schuster KM, Davis KA, Lui FY, Maerz LL, Kaplan LJ. The status of massive transfusion protocols in United States trauma centers: massive transfusion or massive confusion? Transfusion 2010; 50: 1545-51.

12. Patil V, Shetmahajan M. Massive transfusion and massive transfusion protocol. Indian J Anaesth 2014; 58: 590-5.

13. Kashuk JL, Moore EE, Johnson JL, Haenel J, Wilson M, Moore JB, et al. Postinjury life threatening coagulopathy: is 1:1 fresh frozen plasma:packed red blood cells the answer? J Trauma 2008; 65: 261-70.

14. Scalea TM, Bochicchio KM, Lumpkins K, Hess JR, Dutton R, Pyle A, et al. Early aggressive use of fresh frozen plasma does not improve outcome in critically injured trauma patients. Ann Surg 2008; 248: 578-84.

15. Spinella PC, Perkins JG, Grathwohl KW, Beekley AC, Niles SE, McLaughlin DF, et al. Effect of plasma and red blood cell transfusions on survival in patients with combat related traumatic injuries. J Trauma 2008; 64(2 Suppl): S69-77.

16. Gunter OL Jr, Au BK, Isbell JM, Mowery NT, Young PP, Cotton BA. Optimizing outcomes in damage control resuscitation: identifying blood product ratios associated with improved survival. J Trauma 2008; 65: 527-34.

17. Cotton BA, Au BK, Nunez TC, Gunter OL, Robertson AM, Young PP. Predefined massive transfusion protocols are associated with a reduction in organ failure and postinjury complications. J Trauma 2009; 66: 41-8.

18. Schöchl H, Nienaber U, Hofer G, Voelckel W, Jambor C, Scharbert G, et al. Goal-directed coagulation management of major trauma patients using thromboelastometry (ROTEM)-guided administration of fibrinogen concentrate and prothrombin complex concentrate. Crit Care 2010; 14: R55.

19. Pham HP, Shaz BH. Update on massive transfusion. Br J Anaesth 2013; 111 Suppl 1: i71-82.

20. Chaiwat O, Lang JD, Vavilala MS, Wang J, MacKenzie EJ, Jurkovich GJ, et al. Early packed red blood cell transfusion and acute respiratory distress syndrome after trauma. Anesthesiology 2009; 110: 351-60.

21. Acker SN, Partrick DA, Ross JT, Nadlonek NA, Bronsert M, Bensard DD. Blood component transfusion increases the risk of death in children with traumatic brain injury. J Trauma Acute Care Surg 2014; 76: 1082-7.

22. Hill GE, Frawley WH, Griffith KE, Forestner JE, Minei JP. Allogeneic blood transfusion increases the risk of postoperative bacterial infection: a meta-analysis. J Trauma 2003; 54: 908-14

23. Chapman CE, Stainsby D, Jones H, Love E, Massey E, Win N, et al. Ten years of hemovigilance reports of transfusion-related acute lung injury in the United Kingdom and the impact of preferential use of male donor plasma. Transfusion 2009; 49: 440-52.

24. Benson AB, Moss M, Silliman CC. Transfusion-related acute lung injury (TRALI): a clinical review with emphasis on the critically ill. Br J Haematol 2009; 147: 431-43.

25. Whitaker BI, Hinkins S. The 2011 National Blood Collection and Utilization Survey Report. Washington: The U.S. Department of Health and Human Services; 2011. 